\title{
SALUTO DELLA SOPRINTENDENTE ARCHEOLOGIA, BELLE ARTI E PAESAGGIO PER LA CITTÀ METROPOLITANA DI MILANO
}

\author{
ANTONELLA RANALDI (*)
}

Nel portare i saluti del Ministro Dario Franceschini, invitata a partecipare in rappresentanza del MiBACT a questo convegno, ringrazio l'Istituto Lombardo e il comitato scientifico che lo hanno promosso e organizzato.

Il Ministero per i Beni e le Attività Culturali e il Turismo attraversa, in questo momento, una fase di forti cambiamenti, con riforme sostanziali e innovative nell'articolazione dei vari uffici. Si è voluto separare la tutela della valorizzazione, assegnando un maggior peso alle istituzioni museali e incrementandone la fruizione; dall'altra, sono state istituite le Soprintendenze uniche Archeologia, Belle Arti e Paesaggio, deputate alla tutela del patrimonio culturale. Non per questo tutela e valorizzazione sono in contrasto tra loro, come certe volte si sente dire.

I temi all'attenzione di questo convegno negli importanti interventi che seguiranno si riassumono in etica, diritto ed economia dei beni culturali.

Spendo solamente qualche parola di commento. Quanto all'Etica il lavoro all'interno del Ministero è un servizio; mai bisogna pensare che sia esercizio del potere, bensì è esercizio di una funzione. È importante riaffermarlo anche nei ruoli che donne e uomini assumono all'interno dello Stato, con spirito di servizio.

Quanto poi al Diritto, il tema è fondamentale, in quanto la legit-

(*) Soprintendente Archeologia, Belle Arti e Paesaggio per la città metropolitana di Milano, Italia. E-mail: antonella.ranaldi@beniculturali.it 
timità del nostro agire e degli atti prodotti si basa proprio sull'applicazione e chiarezza delle norme, in una materia in cui la componente tecnica e discrezionale di giudizio gioca un ruolo essenziale; l'aspetto anche amministrativo insieme a quello giuridico è un presupposto fondante. Applichiamo una legge, alla quale gli uffici di tutela per primi sono soggetti. Siamo chiamati a dare efficacia e riscontro alle norme del Codice dei beni culturali e paesaggistici secondo i principi di tutela e promozione stabiliti dalla Costituzione (D.Lgs. 42/2004 e smi). Nella tutela i divieti esistono, insieme agli obblighi che spettano in primo luogo a chi ne esercita le funzioni.

Quanto infine all'Economia, parliamo di patrimonio, di beni, di un'eredità che rappresenta un valore, non astratto ma fondato sulla sussistenza e la materialità dei beni stessi. Non credo che la tutela rappresenti una diseconomia. In quanto eredi di un patrimonio, non dobbiamo disperdere la ricchezza di cui siamo depositari, deprezzarla e farle perdere valore, un valore che è sicuramente culturale ma anche economico. La cura e la manutenzione servono a conservare questo patrimonio. Un buon restauro, a cui si accompagnano quasi sempre nuove scoperte e acquisizioni, costituisce incremento di valore in termini sia patrimoniali che culturali. 